\title{
COLORECTAL CARCINOMA STILL A CHALLENGE FOR THE SURGEONS IN 2016
}

\author{
Nikola Kolev \\ Department of General and Operative Surgery, Medical University of Varna
}

Locally recurrent CRC is a disease presenting an exceptional challenge to the surgeon. At the time of the diagnosis local recurrences are often accompanied by complications and engage adjacent organs and anatomical structures. Local recurrences are often associated with systemic ones. Attempts to achieve surgical radicalism performing multivisceral resections in the surgical field of post-operative adhesions, adjuvant RT with neoangiogenesis and dissecting changed plans is often challenging. The presence of residual tumor formation, or transmural lymphatic metastases after primary resection regarding colorectal cancer are the most common cause of local recurrence. As a rule, local recurrences of colorectal cancer are locally advanced - with macroscopic evidence of involvement of adjacent anatomical structures and organs. Usually therapy in these patients is multimodal, requires good communication with the surgeon, the medical oncologist and the radiotherapists, as well as interdisciplinary surgical skills.

Dr. Vesselin Marinov presents two articles on the topic of palliative multivisceral resections for locally advanced colorectal cancer and challenges in the surgical treatment of locally recurrent colorectal carcinoma. Both studies include a patient group with locally recurrent colorectal cancer.

The first study is a retro- and partially prospective analysis of perioperative results for the period of 8 years of multivisceral palliative resections for recurrent colorectal cancer. The results in terms of perioperative morbidity and lethality with an average hospital stay of 8.2 days reported in this publication provide grounds to believe that the removal of the primary tumor in selected patients is the method of choice. Moreover, a combination with an adjuvant chemotherapy improves survival. There are no set rules of surgical behavior in the treatment of locally advanced colorectal cancer. Some studies have reported acceptable levels of perioperative morbidity and mortality rates in selected patients who had undergone resection of a primary tumor. The author supports the thesis that palliative resections prevent obstructive complications, reduce the level of pain and hemorrhage associated with the disease.

In the second article the patients were analyzed by primary location, localization of the recurrence supra- or inframesocolic abdominal cavity. The performed surgeries include palliative and potentially curative resections. Unfortunately, the recurrences of colorectal cancer are usually locally advanced and in a high percentage of cases and accompanied by complications during the diagnosis. The surgical treatment remains the only potentially curative option. Performing multivisceral resections in terms of anatomical changed plans is a challenge for the surgeon. Perianastomotic recurrences without distant dissemination are most favorable for radical surgical treatment. Dr. Marinov concludes that the implementation of en bloc resection without breaking the adherent to the tumor structures is important for the achievement of radicalism. The experience of the surgical team, in the field of pelvic and hepatobiliary surgery, is extremely important. The multimodal approach including chemo- and radiotherapy is especially important in defining the individual strategy.

Scripta Scientifica Medica, vol. 48, No 1,2016, p. 9

Copyright (๑) Medical University of Varna 